\title{
La coordination inter-organisationnelle, levier de la gouvernance territoriale : quelles leçons tirer de la gestion de l'eau interbassin?
}

\author{
Mathieu Dionnet ${ }^{\mathrm{a} *}$ et Lætitia Guérin-Schneider ${ }^{\mathrm{b}}$ \\ ${ }^{a}$ Lisode, 365 rue Jean-François Breton, BP 5095, 34196, Montpellier Cedex 05 \\ 'Institut National de Recherche en Sciences et Technologies pour l'Environnement \\ et l'Agriculture, Irstea, UMR G-EAU \\ 361, rue Jean-François Breton, BP 5095, 34033 Montpellier Cedex 1
}

\section{Résumé}

La gouvernance territoriale met habituellement l'accent sur l'implication des citoyens ou des représentants d'usagers dans la construction de l'action publique. Dans certaines configurations plus complexes, lorsque le problème à résoudre dépasse le territoire ou le domaine de compétence stricte d'un seul acteur public, la gouvernance territoriale passe avant tout par la coordination des différentes organisations ayant une part de responsabilité dans la résolution du problème ou étant impactées par celui-ci. Résultant d'un projet de recherche-intervention sur la gouvernance de l'eau, cet article analyse le cas d'une coordination inter-organisationnelle entre les gestionnaires de deux bassins-versants du Sud de la France partageant les mêmes ressources. En mobilisant une grille d'analyse pluridimensionnelle, nous mettons en lumière les limites de l'action publique territoriale qui entravent aujourd'hui ce type de coordination. Ce travail, renforcé par nos expériences antérieures de conseil auprès des acteurs publics, nous amène à proposer une méthodologie participative qui appuie la coordination inter-organisationnelle. Nous dressons ainsi les contours d'une ingénierie de la gouvernance territoriale dont les modalités restent en grande partie à développer.

Mots clés : gouvernance territoriale, coordination inter-organisationnelle, gestion de l'eau interbassin, SAGE.

(c) 2014 Lavoisier, Paris. Tous droits réservés.

"Auteur correspondant : mathieu.dionnet@lisode.com

doi :10.3166/ges.16.399-420 @ 2014 Lavoisier, Paris. Tous droits réservés. 


\begin{abstract}
Summary
Improving territorial governance through inter-organizational coordination: drawing lessons from inter-basin water management. The notion of "territorial governance" usually focuses on how to involve the public or the stakeholders in decision-making processes related to public issues. In more complex situations, when the problem to be solved goes beyond the area of responsibilities of a single public actor, "territorial governance" relies on the coordination of the different organizations involved in the problem resolution, or impacted by that problem. This study was carried out in an intervention-research project about water governance. Our paper analyses the case of an inter-organizational coordination between the water managers of two river basins from South of France, which share the same resources. By using a multi-dimensional analytical framework, we identify constraints related to local public actions which hamper such coordination. This study, confirmed by previous experiences of consulting services in the public sector, allows us to propose a participatory methodology that promotes inter-organizational coordination. Thus, we intent to outline an engineering of "territorial governance" for which procedures largely remain to be developed.
\end{abstract}

Keywords : territorial governance, inter-organizational coordination, inter-basin water management, SAGE.

() 2014 Lavoisier, Paris. Tous droits réservés.

\title{
Introduction
}

Dans le domaine de la gestion des territoires et des ressources naturelles, l'action publique a subi ces dernières décennies de profondes mutations. La décentralisation, le renforcement du développement territorial et, plus récemment, la démocratie participative, sont autant de processus qui ont conduit l'État à faire évoluer le partage des responsabilités entre les différentes organisations et acteurs du territoire, et plus largement à reconsidérer la manière d'élaborer l'action publique.

Si ces mutations sont communes à de nombreux pays, elles sont néanmoins motivées par différentes logiques, et ce à différentes échelles. À l'échelle d'un État, elles sont souvent la conséquence de politiques libérales d'ajustement structurel prônant un «nouveau management public » qui vise la diminution du pouvoir de l'État, sa décentralisation, l'augmentation de l'influence de la sphère privée, notamment en diffusant ses méthodes de gestion dans la sphère publique, mais également l'augmentation du pouvoir de la société civile. Dans une logique normative, ces politiques ont été largement encouragées par les instances internationales à travers la diffusion du concept de «bonne gouvernance » (Banque Mondiale, 1992). À une échelle plus locale, elles sont davantage la conséquence de politiques qui placent les communautés, les citoyens, les usagers au centre du dispositif de gestion. Leur ambition est alors de mieux partager le pouvoir entre les acteurs locaux et leur encadrement à travers leur participation à la prise de décision sur un sujet les concernant, mais aussi de mieux intégrer les intérêts des différentes parties prenantes. La gouvernance n'a plus à cette échelle d'ambition prescriptive. Au contraire, elle devient un cadre d'analyse des nouvelles formes polycentriques de l'action locale (Stocker, 1998; Petit, 2004; Ghiotti, 2006; Rey-Valette et al., 2011). Elle est associée à des pratiques d'action collective ou à la recherche d'arrangements volontaires formels ou informels. La dimension instrumentale devient alors plus forte (Lardon, 2007; Chia 
et $a l ., 2008)$. Nous pouvons citer en exemples les méthodes participatives appliquées au développement rural de Chambers (1991), les démarches de participation citoyenne développées dans les pays du Nord (Edelenbos, 1999) et du Sud (Baron et Belardi, 2010), les travaux d'Ostrom (1990) et de Petit (2004) sur la gestion des communs, les approches de Borrini-Feyerabend sur la cogestion des ressources naturelles (Borrini-Feyerabend, 2000) ou sur la gouvernance partagée des aires protégées (Borrini-Feyerabend, 2010), les démarches de Gestion Intégrée $\left(\mathrm{GIZC}^{1}, \mathrm{GIRE}^{2} \ldots\right.$ ) (Rey-Valette et Antona, 2010) ou encore l'analyse des contrats de rivières (Brun, 2010).

Aujourd'hui, de nombreux aspects de la vie publique sont ainsi gérés au niveau local, à travers le développement de projets impliquant des acteurs publics (collectivités, services déconcentrés de l'État, Agences de l'Eau...) et des acteurs locaux (représentants d'usagers, société civile, associations, secteur privé...). Ces projets s'inscrivent dans ce que l'on nomme communément la gouvernance territoriale. Traduite dans de nombreux champs de l'action publique, la gouvernance territoriale se décline aujourd'hui en « gouvernance urbaine », "gouvernance des transports », "gouvernance des espaces naturels », « gouvernance de l'eau », etc.

L'étude de ces nouveaux modes d'action publique est nécessaire, tant pour mieux caractériser et comprendre ces mutations, que pour mieux les accompagner. Leur étude n'est cependant pas aisée. D'une part, les champs théoriques à mobiliser pour aborder la gouvernance territoriale renvoient à plusieurs disciplines ${ }^{3}$ dont les cadres et outils d'analyse sont divers. D'autre part, les pratiques à analyser sur le terrain sont multiples et souvent en parfait décalage avec les théories proposées (Pasquier et al., 2007). En se basant sur un cas d'étude dans le domaine de la gestion de l'eau, cet article tente d'apporter un éclairage sur la façon de caractériser et d'appuyer la gouvernance territoriale dans sa dimension inter-organisationnelle. Nous cherchons ainsi à identifier sur quoi se joue concrètement la gouvernance territoriale dans un cas concret, quelles sont les contraintes en jeu (structurelles et humaines) et les leviers d'action, et enfin les mécanismes et processus qui permettent de lever certaines de ces contraintes.

Cette recherche a été conduite dans le cadre du projet InterSAGE ${ }^{4}$, sur un territoire recoupant deux bassins-versants littoraux méditerranéens (vallée de l'Orb et du Libron, et Basse Vallée de l'Aude). Du fait de la demande de certains gestionnaires de ce territoire de faire évoluer la gouvernance en place, ce projet s'est inscrit dans une démarche de rechercheintervention (David, 2008). Son objectif était donc double : générer des connaissances sur la gouvernance de l'eau tout en engageant les organisations partenaires dans un processus de changement. Nous présentons tout d'abord notre cadre d'analyse de la gouvernance qui croise différentes dimensions, ainsi que la méthodologie déployée sur le terrain. En appliquant ce cadre, nous analysons ensuite le cas d'étude interSAGE, qui révèle autant

\footnotetext{
${ }^{1}$ Gestion Intégrée des Zones Côtière

${ }^{2}$ Gestion Intégrée des Ressources en Eau

${ }^{3}$ Notamment sciences politiques, gestion publique, économie, géographie et droit, comme l'illustrent les citations faites ci-dessus dans l'introduction et ci-dessous au point 2.1 .

${ }^{4}$ Le projet InterSAGE (2010-2011) a bénéficié du soutien financier du Ministère de l'Ecologie (programme LITEAU), de l'Agence de l'eau Rhône Méditerranée et Corse, du Conseil Régional Languedoc Roussillon et du Conseil Général de l'Héraut.
} 
la nécessité que la difficulté qu'éprouvent les organisations concernées à se coordonner en dehors des cadres réglementaires habituels. De cette expérience, nous extrapolons un certain nombre de recommandations pour faciliter la coordination entre différentes organisations en situation de gouvernance territoriale. Nous tentons donc d'apporter des réponses génériques aux questions relatives à l'organisation du dialogue et à la manière de construire des décisions communes entre des organisations aux intérêts divers. Cet article dresse ainsi les contours d'une nouvelle forme d'ingénierie qui répond aux enjeux de la gouvernance territoriale décrits par Chia et al., (2008 p. 174) comme « (...) la production d'un langage commun, d'un projet commun, des règles qui permettent aux acteurs de mettre en place des processus d'apprentissages qui favorisent l'action commune.».

\section{Méthodologie}

\subsection{De la gouvernance territoriale à la coordination inter-organisationnelle}

Le concept de gouvernance revêt différents sens lorsqu'il s'applique à la construction de l'action publique (Pasquier et al., 2007; Chia et al., 2008). Les origines de ce terme, tout comme sa finalité, appartiennent à des écoles de pensées différentes, parfois opposées, et génèrent des débats dans la littérature (Leloup, 2005; Froger, 2006). Tandis qu'en sciences de gestion, certains évoquent un modèle décloisonné et intégratif conférant à ceux qui le mettent en place certains avantages par rapport aux autres (Edelenbos et Teisman, 2011), les sciences politiques traduisent plutôt la gouvernance comme le passage d'un modèle centralisé et descendant à un modèle territorialisé et participatif (Leloup, 2005). Plusieurs raisons sont apportées pour comprendre ce renouveau de l'action publique. Dans l'approche systémique, on estime que la gouvernance territoriale est la conséquence de la complexité des questions que l'État doit résoudre, questions qui impliquent de plus en plus d'acteurs dont les intérêts sont interconnectés (Pasquier et al., 2007; Edelenbos et Teisman, 2011). Dans une vision plus postmoderne, la gouvernance traduit plutôt la perte de légitimité de l'État, qui peine tant à représenter l'intérêt général qu'à mobiliser une expertise valable (Duran et Thoenig, 1996; Chevalier, 2003; Lascoumes, 1990). Enfin, une analyse plus marxiste présente la gouvernance comme le souhait des détenteurs du pouvoir d'instaurer de nouvelles formes de contrôle social (Dufour, 2009), en l'assimilant à une autorégulation des intérêts privés.

Un consensus semble néanmoins apparaître sur le fait qu'une coordination centrale ne peut pas résoudre tous les problèmes. Elle doit être complétée par des modes de coordination plus horizontaux, souples, dynamiques, intégratifs, qui remettent en question les cloisonnements, les compétences et le fonctionnement des organisations existantes (Edelenbos et Teisman, 2011), mais aussi les fondements de la démocratie représentative (Pasquier et al., 2007; Blondiaux 2008). De nombreux travaux portent ainsi aujourd'hui sur la place et le rôle des acteurs locaux, notamment des citoyens dans cette nouvelle forme d'action publique (Blondiaux, 2008; Rowe et Frewer, 2000). La gouvernance territoriale s'incarne alors à travers des processus de concertation, lors desquels des propositions sont co-construites entre divers acteurs locaux et leur encadrement. Dans cette configuration, le pouvoir de décision reste au final entre les mains des institutions issues de la démocratie représentative. C'est d'ailleurs bien là que réside la différence entre la concertation et la délégation de pouvoir. 
Dans une telle situation de gouvernance territoriale, un acteur public mène une concertation avec un certain nombre d'acteurs locaux concernés, clairement identifiables, sur une question ou un problème bien cerné dont il porte la responsabilité (p.ex. concertation sur le développement d'un plan local d'urbanisme par une commune, sur le schéma départemental de transport par un Conseil Général, etc.). Divers travaux analysent ces processus de concertation ou proposent des outils pour organiser le dialogue entre les différents acteurs (voir par exemple les guides de Barret (2003) ou de Creighton (2005)).

La situation se complique lorsque le problème dépasse les périmètres de compétences strictes d'un acteur public, sans pour autant être précisément cadré au niveau réglementaire. Dans ce cas, la gouvernance territoriale ne repose pas tant sur l'implication des acteurs locaux à la prise de décision, mais plutôt sur la coordination entre les différentes organisations concernées et responsables d'une partie du problème (et qui peuvent potentiellement avoir des objectifs en compétition). Ces organisations doivent, en effet, considérer les intérêts et actions des autres avant de faire leurs choix, dans la mesure où ceux-ci sont interdépendants. Nous pouvons ainsi émettre l'hypothèse que dans une telle situation, la gouvernance territoriale passe avant tout par une meilleure coordination interorganisationnelle, objet que notre travail tente d'éclairer. Cette hypothèse se distingue de la posture classique qui consiste à mettre l'accent sur le développement de la participation du public. La thèse soutenue ici est que, dès que le problème à gérer dépasse le périmètre d'une seule organisation, il est tout aussi important de travailler sur leur coordination.

Si les travaux qui abordent la gouvernance territoriale y compris dans une dimension instrumentale et opérationnelle sont de plus en plus nombreux (Rey-Valette et al., 2011; Moquay et al., 2001; Lardon, 2007), rares sont ceux qui abordent cette problématique à travers sa dimension inter-organisationnelle (Vanier 2008). En effet, les travaux qui abordent le problème de la coordination des organisations appartiennent tout d'abord aux sciences de gestion, qui s'intéressent à la façon de renforcer l'efficience, la compétitivité, les bénéfices d'entreprises qui trouvent ainsi un avantage mutuel à collaborer (Schmitt et al., 2005; Ring et Van de Ven, 1994; Cattellin, 2008; Xhauflair et al., 2010), comme c'est le cas dans les districts industriels (Becattini, 1989). Ils appartiennent ensuite aux sciences politiques et à la sociologie des organisations qui analysent les coordinations sous l'angle des jeux de pouvoir en place (Crozier et Friedberg, 1977 ; Faure et Negrier, 2007). Il apparaît donc nécessaire de concevoir une grille d'analyse capable d'aborder les différentes dimensions de la coordination inter-organisationnelle.

\subsection{Une grille d'analyse pluridimensionnelle}

La coordination inter-organisationnelle s'opère donc entre des organisations autonomes qui sont impliquées, à divers degrés, dans la gestion d'une ressource ou d'un problème d'intérêt général qu'elles ne maîtrisent pas en totalité. Dans ce contexte, le pouvoir et la légitimité à agir sont donc partagés entre plusieurs acteurs qui ne sont pas liés par un quelconque lien de subordination. Pour comprendre comment des acteurs, indépendants du point de vue de la subordination formelle, peuvent agir ensemble, la question clef consiste à trouver ce qui fait lien entre eux. Dans cette optique, plusieurs dimensions doivent être croisées pour comprendre mais aussi appuyer le développement de coordinations. 
Le lien peut d'abord se construire autour du contexte physique, notamment, pour la gestion de l'eau, autour de dépendances hydrauliques. Il convient alors d'analyser l'origine de l'eau et les transferts entre les organisations. Mais le lien se joue aussi à d'autres niveaux.

Ainsi, dans une optique s'inscrivant dans l'école de la régulation (Jessop, 1998), Baron (2003) affirme que la gouvernance renvoie à une forme de coordination qui se joue à trois niveaux : « des réseaux interpersonnels auto-organisés basés sur la confiance et la réciprocité, une coordination « inter-organisation négociée » qui suppose des formes de partenariat entre des organisations dotées par ailleurs d'une certaine autonomie, et enfin une coordination à un niveau « inter-systémique » ». Le niveau « inter-systémique » désigne ici la coordination entre des systèmes institutionnels qui développent des valeurs et des règles qui leur sont propres et qui ne sont pas forcément partagées en dehors du système. Par exemple, le monde de la gestion de l'eau et de la protection des milieux aquatiques constitue un système institutionnel distinct de celui de l'urbanisme et du développement économique. Le niveau intersystémique met donc en jeu les coordinations entre des ordres institutionnels spécifiques qui sont généralement relativement autonomes les uns par rapport aux autres en termes de référent réglementaire, de partenariat mis en place et de valeurs partagées. C'est typiquement le cas entre le système de la gestion de l'eau et celui de la gestion du territoire, dont les intérêts sont parfois en contradiction, mais qui néanmoins entretiennent des liens (Narcy, 2000).

Outre la dépendance physique, il semble donc pertinent de considérer les relations entre individus, les dispositifs de coordinations formels entre organisations et enfin la coordination institutionnelle. Ces éléments sont en partie dépendants les uns des autres. Les individus font partie d'organisations qui structurent les liens entre personnes. Les systèmes institutionnels sont adoptés par les individus et, dans une certaine mesure, par les organisations dont les procédures et la structuration interne sont marquées par les normes institutionnelles (Meyer et Rowan, 1977).

Comment structurer ces différents types de coordination pour aboutir à une grille d'analyse qui permette de porter un diagnostic opérationnel sur la gouvernance en place, de trouver ses forces et ses limites, et d'identifier des leviers d'amélioration ? Pour répondre à cette question, l'approche développée pour la recherche-intervention par le Centre de Gestion Scientifique (CGS) de l'Ecole des Mines de Paris paraît pertinente (Riveline, 1991). Elle s'appuie sur une grille d'analyse qui vise à appréhender le terrain pour identifier des leviers de changement. Bien que développée pour le contexte de l'entreprise, a priori assez différente de celui de la gouvernance territoriale, sa finalité est proche de celle qui nous intéresse ici : comprendre et faire évoluer la coordination des actions de différents acteurs pour une meilleure cohérence des décisions collectives. Le CGS propose d'identifier quatre niveaux auxquels tout paramètre de gestion peut se rattacher : (i) la matière, qui est considérée comme tout ce qui ne peut être discuté, (ii) les personnes, considérées comme ce qui peut changer quand on remplace un individu, (iii) les institutions, vues comme l'ensemble des comportements permis, interdits ou obligatoires, étant formalisés, (iv) le sacré, c'est-à-dire les normes culturelles (règles informelles et valeurs). Les dysfonctionnements dans la coordination et les décisions collectives peuvent alors bien souvent s'interpréter comme l'apparition d'une incohérence entre ces niveaux. Il se crée des décalages entre des logiques locales qui génèrent des effets pervers.

Nous proposons une synthèse de ces différentes considérations sous la forme d'une grille d'analyse de la gouvernance privilégiant quatre dimensions : 
1. Le contexte physique qui conditionne ce qui est matériellement possible (p.ex. pour la gestion de l'eau, origines de l'eau, infrastructures de transferts, volumes en jeu...).

2. L'environnement institutionnel (règles formelles et informelles, normes, valeurs, coutumes...) qui conditionne la manière dont les acteurs s'autorisent à agir.

3. Les acteurs, individus ou organisations, impliqués dans les actions.

4. Les mécanismes de coordinations entre les acteurs, définis à travers leur finalité (planification, action, arbitrage, contrôle...), la composition des acteurs impliqués et la nature des liens (cf. supra les trois formes de coordinations identifiées par l'école de la régulation, Théret, 1998).

La dimension historique vient compléter ces quatre dimensions. Son intérêt dans une approche portant sur la gouvernance a été souligné par Papadopoulos (2003). Elle révèle des dépendances de sentier et des inerties à la fois matérielles (des infrastructures construites dans le passé conditionnent les transferts d'eau actuels) et cognitives (certains acteurs ont pris l'habitude d'intervenir ou non dans la gestion de l'eau).

Comme pour la grille du CGS, la capacité à porter un diagnostic de la gouvernance vient de la confrontation entre ces dimensions. Elle résulte de la recherche de cohérence entre les logiques sous-tendent ces différentes dimensions : est-ce que les acteurs et organisations dépendantes physiquement sont liés par des mécanismes de coordination? Existe-t-il des règles sur les transferts d'eau?

Cette grille opérationnalise la gouvernance dans la mesure où elle décrit des dimensions explicites et précises et parce qu'elle est tournée vers le diagnostic et in fine vers la reconfiguration des coordinations en place. L'objet du présent article n'est pas de rentrer plus avant dans les considérations méthodologiques sur la construction et l'utilisation de cette grille, mais de livrer les principaux résultats qui découlent de son utilisation. Les aspects liés à la grille en tant qu'innovation méthodologique font l'objet d'un article spécifique en cours d'écriture.

Mobilisée sur le terrain du projet InterSAGE, cette grille a permis de réaliser un diagnostic participatif de la gouvernance en place. La méthodologie a reposé sur : (i) la conduite et l'analyse de 47 entretiens individuels au sein d'organisations impliquées dans la gestion de l'eau dans l'Ouest Hérault (services déconcentrés de l'État, collectivités territoriales, syndicats mixtes, établissements publics, etc.) et d'usagers de l'eau (agriculteurs, gestionnaires des services d'eau potable, campings, etc.); (ii) la réalisation d'un atelier de modélisation participative ${ }^{5}$ sur la gestion de l'eau dans l'Ouest Hérault réunissant 16 gestionnaires locaux (Guérin-Schneider et al., 2010); (iii) la récolte et l'analyse de bases de données et de documents administratifs (données sur le suivi des ressources et des usages, réglementation, contrats de délégation de services d'eau potable, etc.); et enfin (iv) l'animation et l'évaluation de trois ateliers participatifs réunissant 27 participants incluant des représentants d'usagers, des agents des organisations publiques et des élus et utilisant un jeu de rôles baptisé SIMUL'EAU ${ }^{6}$, dont l'objectif était de mener une réflexion collective sur la façon

${ }^{5}$ Un exercice de modélisation participative est une méthode permettant à un groupe de participants de développer, en commun, un modèle qui correspond à la représentation que se fait le groupe d'une situation, d'un problème ou d'un système.

${ }^{6}$ Un film présentant SIMUL'EAU est accessible sur le lien suivant : http://www.youtube.com/watch?v= hOhx671p868\&feature=youtu.be 
d'améliorer la gouvernance en place. Ce diagnostic a permis de caractériser puis de croiser les différents niveaux d'analyse afin de faire ressortir les limites de la gouvernance en place et les besoins de nouvelles coordinations entre organisations.

\section{L'Inter-SAGE Orb - Basse Vallée de l'Aude : enjeux et limites d'une coordination inter-organisationnelle}

2.1. Une gouvernance mise à l'épreuve par un transfert d'eau entre deux bassins-versants

Les grands principes, définis au niveau national ou supranational et formalisés dans la réglementation, se heurtent souvent à la complexité des situations locales. La gestion de l'eau est un domaine particulièrement riche pour illustrer ce constat. Nous analysons ici l'exemple du transfert d'eau entre le bassin-versant de l'Orb et celui de la Basse Vallée de l'Aude. Cette analyse permet de mettre en évidence les difficultés rencontrées par les gestionnaires pour mettre en place des modes de coordination efficaces et légitimes.

En France, la gouvernance de l'eau est cadrée par un contexte législatif qui repose sur le principe de subsidiarité, notamment par une gestion à l'échelle des bassins-versants (Barraqué, 1997). La réglementation européenne ${ }^{7}$, retranscrite au niveau national ${ }^{8}$, prévoit la définition d'un Schéma Directeur d'Aménagement et de Gestion des Eaux (SDAGE) au niveau des grands districts hydrographiques qui encadrent, au travers de grands principes, la mise en œuvre du Schéma d'Aménagement et de Gestion des Eaux (SAGE) au niveau des sous-bassins ou bassins intermédiaires. Le SAGE est l'échelle à laquelle doivent se définir les conditions de réalisation des objectifs de préservation des milieux aquatiques, mais aussi se prendre les décisions concernant la répartition de la ressource entre les différents usages, et leur priorisation. Les SAGE sont pilotés par des Commissions Locales de l'Eau (CLE) composées pour moitié d'élus, pour un quart de représentants des usagers de l'eau et pour un quart de représentants des services de l'État (Latour et Lebourhis, 1995). En complément, des règles spécifiques peuvent être élaborées par les autorités préfectorales, comme l'arrêté cadre sécheresse.

Comme décrit dans le SDAGE Rhône Méditerranée et Corse (2010-2015), certaines configurations requièrent la mise en place d'une démarche supra bassin. C'est le cas lorsqu'un bassin-versant transfère une partie de son eau à un autre bassin. Dans ce cas, des «complémentarités entre démarches de gestion locale par bassin-versant et approches supra bassin doivent ainsi être trouvées. (...) À ce titre, les «bassins émetteurs » et les «bassins récepteurs » doivent se coordonner pour une meilleure gestion de la ressource ${ }^{9} »$. Ainsi, bien que le pouvoir de gestion (notamment le partage de la ressource en eau entre usages) soit normalement confié aux SAGE dont le découpage est hydrographique, dans la pratique, il doit parfois se construire entre plusieurs organisations partageant un même bassin fonctionnel ${ }^{10}$.

\footnotetext{
${ }^{7}$ Directive Cadre Européenne sur l'Eau (n² 2000/60 du 23 octobre 2000)

${ }^{8}$ Loi du 21 avril 2004 \& loi sur l'Eau et les Milieux Aquatiques du 30 décembre 2006

${ }^{9}$ Disposition 4-03 SDAGE RM\&C 2010-2015

${ }^{10}$ La notion de bassin fonctionnel s'oppose à celle de bassin-versant, en ce sens qu'elle ne correspond pas uniquement à la portion de territoire délimitée par des lignes de crête dont les eaux alimentent un exutoire commun, mais intègre également les autres territoires qui seraient hydrologiquement liés.
} 
C'est le cas pour le bassin-versant de l'Orb et du Libron, dont les ressources et les usages sont schématisés dans la figure 1 (voir page suivante). Dans ce dernier, entre 50 et 55 millions de $\mathrm{m}^{3}$ d'eau sont prélevés chaque année dans le milieu ${ }^{11}$, tous usages confondus, principalement dans le fleuve Orb (40-45 $\left.\mathrm{Mm}^{3}\right)$, mais aussi dans le Canal du Midi approvisionné par le fleuve Aude (environ $6 \mathrm{Mm}^{3}$ ) et la nappe de l'Astien (environ $3 \mathrm{Mm}^{3}$ ). Une part importante des prélèvements effectués sur l'Orb est cependant transférée sur le territoire audois (environ $20 \%$ ). Il sert à l'alimentation en eau potable des communes littorales touristiques audoises (environ $5 \mathrm{Mm}^{3}$ ), et aux périmètres irrigués à cheval entre les départements de l'Hérault et de l'Aude $\left(15 \mathrm{Mm}^{3}\right)$. Agé de plus de 40 ans, ce système de transfert a été mis en place par l'État à travers la Mission dite Racine ${ }^{12}$. Le barrage des Monts d'Orb et la station de pompage de Réals ont été construits pour assurer ce transfert: l'eau stockée l'hiver est relâchée en été pour être ensuite transférée sur le bassin audois dont les ressources sont faibles et sans commune mesure avec l'afflux de population estivale que connaît la zone. En pratique, le même opérateur $\left(\mathrm{BRL}^{13}\right)$ gère le barrage et vend l'eau transférée via Réals aux communes audoises. Cet opérateur dispose d'un droit de prélèvement accordé par un arrêté préfectoral de 1969 qui impose deux conditions : respecter un débit total maximum de prélèvement, et respecter un débit minimum dans l'Orb à l'aval de la prise d'eau.

Le panorama institutionnel en lien avec ce transfert comprend donc deux SAGE sur deux départements, ainsi qu'une structure de droit privé dont le champ d'action est encadré par des collectivités territoriales et l'État. En 2013, les deux SAGE de l'Orb et de la Basse Vallée de l'Aude sont toujours en cours d'élaboration. Ils doivent rapidement définir les volumes maximums prélevables ${ }^{14}$ ainsi que la répartition de ces volumes entre usages. En pratique, des commissions thématiques sont organisées et animées par les équipes techniques des syndicats porteurs ${ }^{15}$ des SAGE, afin de construire des propositions qui sont par la suite validées par les CLE. Si cette procédure est en théorie très bien structurée, le transfert entre deux bassins brouille les cartes. En effet, bien que ce cas ait été envisagé au niveau du SDAGE, aucune procédure formelle n'est proposée pour assurer la cohérence entre deux SAGE qui encadrent l'usage de ressources partagées.

${ }^{11}$ D'après les résultats du projet InterSAGE (2009-2011) ainsi que le diagnostic SAGE Orb-Libron (2013).

${ }^{12}$ La mission interministérielle d'aménagement touristique du littoral du Languedoc-Roussillon est une structure administrative française créée en 1963 par l'État pour conduire de grands travaux d'infrastructure en vue de développer le littoral de la Méditerranée sur les $240 \mathrm{~km}$ de côtes qui vont du petit Rhône à la frontière espagnole.

${ }^{13}$ BRL Exploitation, Société Anonyme à conseil d'administration appartient au groupe BRL, lui-même Société d'Économie Mixte Locale, société régie par le droit privé associant dans son capital des collectivités territoriales à hauteur de 75,6 \% (majoritairement la Région Languedoc-Roussillon et les départements du Gard, de l'Héraut et de l'Aude) ainsi que d'autres actionnaires (Caisse des Dépôts et Consignations, Caisse d'Épargne, Crédit Agricole, etc.).

${ }^{14}$ Le volume maximum prélevable par l'ensemble des usagers d'un bassin ou d'un aquifère est le volume qui permet de satisfaire, 8 années sur 10, les débits d'objectif d'étiage et/ou l'équilibre quantitatif de la masse d'eau souterraine. Il prend en compte les ressources en eau naturelles et stockées (loi sur l'eau du 30 déc. 2006).

${ }^{15}$ Dans notre cas, le Syndicat Mixte du Bassin-Versant de l'Orb et du Libron et le Syndicat Mixte du Delta de l'Aude. 
Figure 1 : Schéma synthétisant les différents usagers Héraultais (en gris foncé) et Audois (en noir) ainsi que leur approvisionnement en eau (par l'Orb, l'Aude, le Canal du Midi et la nappe astienne). On observe la complexité du réseau qui interconnecte les deux bassins-versants.

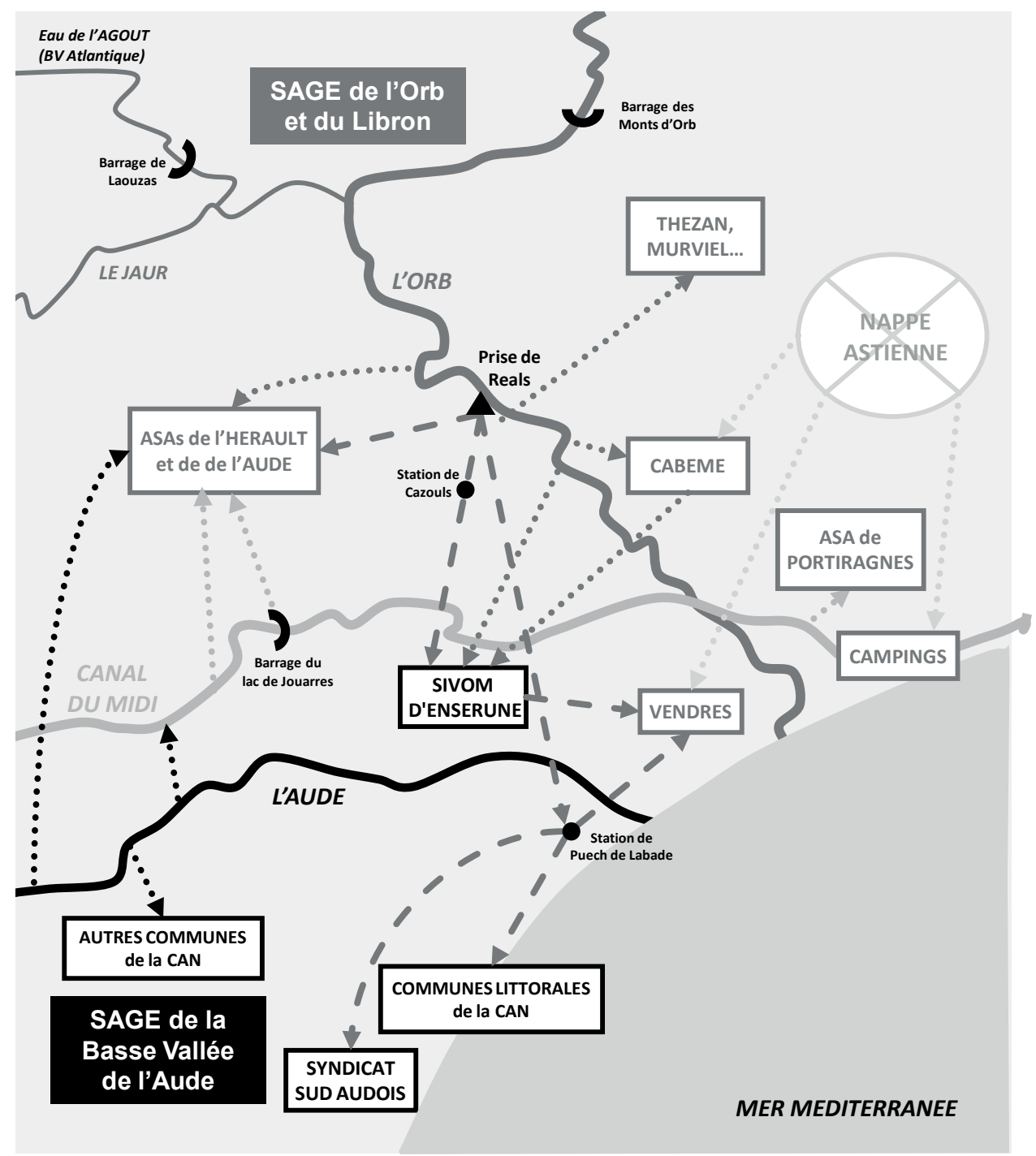


Or, l'enjeu est de taille. Dans ce contexte littoral caractérisé par une économie du tourisme importante, une profession agricole en crise à la recherche d'alternatives à la viticulture traditionnelle (notamment grâce à l'irrigation), des villes qui s'accroissent plus vite que la moyenne nationale et des écosystèmes menacés, le partage de l'eau est hautement stratégique. À la concurrence entre usages et entre territoires, viennent se rajouter les rivalités entre élus de différents bords et aux différents types de mandats politiques ${ }^{16}$.

Pour ne pas voir l'État arbitrer le partage de leurs ressources, les gestionnaires de bassins doivent donc interagir de façon à définir, ensemble, les conditions d'une gestion supra SAGE communément appelée «l'Inter-SAGE ». Aujourd'hui, de nombreuses initiatives locales sont en train de voir le jour. Certaines sont déjà formalisées (Comité Technique InterSAGE ${ }^{17}$ de l'Aude, Aqua Domitia $^{18}$ ) alors que d'autres restent à un stade informel (rencontres entre les CLE de l'Orb et de la basse vallée de l'Aude, réunions entre les directeurs des structures porteuses de ces SAGE). Cependant, notre diagnostic, appuyé sur la confrontation des quatre dimensions de la grille définie ci-dessus, révèle que ces initiatives se heurtent à certaines caractéristiques du fonctionnement de l'action publique territoriale qui entravent la coordination des organisations concernées.

\subsection{Des limites à la coordination entre les acteurs publics}

\subsubsection{Difficile adéquation entre les limites administratives et la réalité physique des ressources gérées}

Le croisement de l'analyse du contexte physique avec les mécanismes de coordinations entre acteurs (dimension 1 et 4 de la grille proposée) permet de mettre en relief les interdépendances matérielles et de les comparer aux coordinations existantes. Il apparaît tout d'abord que les interactions entre les organisations ne sont pas forcément cohérentes avec les continuités hydrologiques les liant objectivement. En effet, la réglementation, que le Préfet a choisi d'appliquer à la lettre, demande que les acteurs représentés dans les CLE soient ceux du périmètre du SAGE. Les syndicats de bassin et les collectivités bénéficiant du transfert d'eau de l'Orb vers la Basse Vallée de l'Aude sont alors exclus de la CLE de l'Orb, et inversement pour la CLE de la basse vallée de l'Aude. La transversalité entre les SAGE de bassins-versants distincts mais interconnectés reste donc limitée. Les structures de bassin, conscientes des enjeux qui les lient, s'invitent cependant réciproquement aux commissions thématiques et aux CLE de manière informelle.

La frontière administrative départementale est encore plus marquée que la frontière naturelle du bassin-versant. Les services de l'État se sont organisés en Mission InterServices de l'Eau (MISE) afin de faire examiner, par l'ensemble des services de l'État, les dossiers et de rendre le plus rapidement possible un avis ou une décision, mais leur

\footnotetext{
${ }^{16}$ Parmi les acteurs clés du territoire on compte des maires, des Présidents d'Agglomération, des Conseillers Généraux, des Députés et des Sénateurs.

${ }^{17}$ Comité restreint répondant à une injonction du Préfet coordonnateur de bassin d'engager « la concertation relative aux transferts d'eau avec les bassins-versants voisins » afin d'assurer une cohérence des politiques de l'eau.

${ }^{18}$ Instance de concertation créée par arrêté préfectoral en janvier 2009. Ses objectifs s'appuient sur les orientations du SDAGE et de la charte de gestion durable de l'eau (élaborée par le Conseil Régional) qui préconisent la création d'instances de gestion concertée de la ressource à un niveau supra bassin cohérent.
} 
périmètre d'action reste confiné au département. Ceci pose notamment problème pour les arrêtés sécheresse qui ne prévoient pas systématiquement d'appliquer aux communes du département, alimentées par une ressource venant d'un autre département, les mesures de restriction prises sur cette ressource limitrophe. Ainsi, en cas de sécheresse, certaines communes du bassin-versant de l'Orb se sont vues imposer des restrictions, tandis que les communes audoises desservies par la même ressource ne mettaient en place aucune mesure $^{19}$. À nouveau, certains arrangements informels voient néanmoins le jour afin d'ajuster la mise en œuvre des mesures de restriction aux réalités du terrain. Ainsi les animateurs des MISE audoise et héraultaise tentent de se tenir informés de leurs décisions pour répercuter d'un département sur l'autre les mesures prises.

Ce constat illustre bien comment les limites administratives peuvent freiner la mise en place d'une action publique territoriale cohérente. Dans un contexte où le territoire administratif n'englobe pas le système fonctionnel complet, et où personne n'a le pouvoir d'agir sur la totalité du système, de nouveaux modes d'organisation sont nécessaires pour construire une vision partagée de l'ensemble du système et permettre de prendre des décisions et entreprendre des actions à plusieurs.

\subsubsection{Déficit de liens formels entre outils de planification et outils de mise en ouvre}

La confrontation de l'analyse des règles formelles qui caractérisent une partie de l'environnement institutionnel avec les acteurs (dimensions 2 et 3 de la grille) révèle une mauvaise articulation entre les différents niveaux de gestion. Si l'on se réfère à la réglementation, le SAGE est un outil de planification et d'encadrement des usages de l'eau sur un bassin donné, orchestré par une commission représentant élus, usagers et pouvoirs publics. Il s'agit donc, en théorie, de l'instrument de pilotage de la gestion de l'eau sur les territoires. Mais, les actions de gestion se situant au niveau le plus bas sont effectuées par des opérateurs de réseaux d'eau brute et d'eau potable. Notre analyse révèle en effet que les syndicats rencontrés ne prévoient pas, à l'heure actuelle, de mécanisme formel permettant d'intervenir dans la formulation des mandats fixant les obligations de ces opérateurs en termes de prélèvement dans les ressources.

Ainsi, lorsque l'opérateur du réseau d'eau brute décide de lâcher de l'eau du barrage ou de transférer des volumes vers les communes audoises, il n'est en pratique soumis qu'au respect de l'arrêté préfectoral conditionnant le débit à laisser à l'aval de la prise d'eau. Une fois cette condition remplie, ses choix sont ensuite régis par des contrats qui le lient à ses clients, et qui lui imposent de fournir un certain volume d'eau. Les délégataires des réseaux d'Alimentation en Eau Potable sont, quant à eux, soumis au respect des contrats de délégation signés avec les communes ou leur regroupement, mais pas avec les syndicats en charge des SAGE. Sur notre périmètre, ces derniers n’ont généralement pas été consultés lors de la signature des contrats de délégation. Les contrats étudiés se révèlent ainsi extrêmement succincts sur les mesures de régulation et les arbitrages entre ressources à mettre en œuvre par les opérateurs ayant accès à plusieurs ressources interconnectées. Jusqu'à présent, les contrats abordaient les mesures opérationnelles pour faire face au risque de pénurie et anticiper la crise. Le fait que l'Orb n'ait jamais connu de

\footnotetext{
${ }^{19}$ En revanche, l'arrêté sécheresse du 4 avril 2007 de l'Hérault prévoit bien la prise en compte des mesures de restriction des départements limitrophes pour les communes héraultaises bénéficiant de transferts inter-départementaux.
} 
crise majeure jusqu'à présent est un élément d'explication, mais sur d'autres zones où des contrats ont pu être observés, ce manque de coordination entre planification et contrats opérationnels est également observé.

\subsubsection{Des tensions entre des acteurs légitimes mais aux visions politiques divergentes}

La comparaison des valeurs sous-tendant les positions des différents acteurs (dimension 2) permet de révéler des divergences politiques. Pour les acteurs audois, tout comme pour les représentants de la Région Languedoc Roussillon (LR) et et de l'État, le transfert se justifie par le principe que l'on peut qualifier de «solidarité interbassin ». Le littoral audois est si mal doté en ressources que son développement repose en grande partie sur les ressources des bassins-versants limitrophes. Pour les acteurs biterrois en revanche, l'eau est perçue comme un «bien territorial » dont le partage doit se faire localement au sein du bassin-versant car il oriente les développements territoriaux locaux possibles. Le président de l'agglomération de Béziers, en place jusqu'en 2013, interpelle ainsi régulièrement le Conseil Général de l'Aude et la Région LR pour faire valoir son droit de subsidiarité.

Plus généralement, se pose alors la question de la superposition des légitimités démocratiques des différentes organisations impliquées dans la planification de l'action publique territoriale (p.ex. entre une Commune, une Agglomération, un Conseil Général ou une Région). Comme décrit par Ghiotti (2006), la décentralisation s'est soldée par « une sévère compétition entre institutions pour l'appropriation et le contrôle du ou d'un territoire, vu comme un moyen d'asseoir leur politique, d'affirmer leur légitimité et leur existence ». Chaque niveau de gestion peut légitimement prétendre que sa vision reflète l'intérêt général, engendrant de ce fait de nombreuses tensions. Dans ce contexte, des mécanismes de régulation sont nécessaires afin de «sortir des coulisses de l'informel » et de rendre transparents les processus de négociation et les arbitrages ou choix qu'ils engendrent.

\subsection{Un besoin d'accompagnement spécifique}

L'approche consistant à croiser les différentes dimensions de notre grille d'analyse a permis d'identifier certains freins qui limitent la coordination des acteurs concernés. Il s'agit de contraintes structurelles liées à l'environnement physique et au système de gestion en place (comme démontré dans les paragraphes 3.2.1 et 3.2.2), mais aussi de tensions provenant des individus et des valeurs qu'ils portent (paragraphe 3.2.3). Or, notre analyse révèle que ces différents freins n'agissent pas forcément avec la même intensité sur tous les acteurs rencontrés. En effet, leurs capacités à faire face à ces contraintes, notamment les marges de manœuvre pour innover, varient énormément. Ceci découle de différences liées à l'environnement institutionnel et aux personnes qui s'y situent?

Tout d'abord, le fonctionnement interne des organisations change d'une structure à une autre. La hiérarchie interne, le cloisonnement entre services, le turnover, la relation avec les organes de tutelle (élus pour les collectivités, ministères pour les services de l'État) sont autant de facteurs qui contribuent ou entravent la création de relations entre des individus dont le champ de compétence professionnelle strict ne leur impose pas de se rencontrer. Le partage d'un même système institutionnel (Narcy, 2000) facilite grandement la création de ces relations, dans la mesure où les personnes concernées reconnaissent les 
mêmes règles et partagent le même vocabulaire ainsi que les mêmes références. Les gestionnaires du bassin de l'Orb éprouvent ainsi plus de facilité à communiquer avec leurs homologues audois qu'avec les gestionnaires urbains de leur territoire.

Ensuite, les personnes engagées dans une coordination ne se situent pas forcément sur le même pied d'égalité. D'une part, tout engagement dans une démarche non formalisée représente un risque qui ne sera pas apprécié de la même façon d'un individu à un autre. N'étant pas égaux dans leur capacité à communiquer leurs idées ou défendre leurs intérêts, certains « risquent» plus que d'autres. D'autre part, le pouvoir d'action ou de décision n'est pas le même pour chaque acteur. Pour les élus ou chefs de service, le risque à s'engager dans une nouvelle configuration est faible, dans la mesure où cette décision est de leur responsabilité. Pour les techniciens en revanche, toute proposition n'ayant pas été antérieurement validée par leur tutelle ou leur hiérarchie peut les mettre en danger. Gérer ces asymétries de pouvoir et de capacité à s'exprimer nécessite des compétences particulières.

Ainsi, si les gestionnaires de l'Orb et de la Basse Vallée de l'Aude se coordonnent, c'est parce que leur environnement institutionnel le leur permet, mais aussi parce qu'ils en ont individuellement la capacité. Réunir ces conditions est d'autant plus difficile que le nombre d'organisations est grand et les systèmes institutionnels variés. Dans ce cas, la plupart des personnes interviewées s'accordent sur la nécessité de mobiliser un accompagnement spécifique.

Or, il n'existe actuellement pas d'ingénierie de la coordination inter-organisationnelle en appui aux acteurs publics (Vanier, 2008; Moss, 2009). En effet, les prestations de conseil habituelles se limitent souvent à de la maîtrise d'œuvre technique, de l'assistance juridique et financière ou de l'assistance à la concertation locale. Ces prestations sont conçues par et pour une structure confrontée à un problème de sa compétence (champs d'action) dans le cadre d'une procédure administrative sous sa seule responsabilité. Au contraire, un problème de coordination inter-organisationnelle nécessiterait une prestation pilotée par (et destinée à) plusieurs organisations légitimes, sans procédure prédéfinie. Dès lors il convient de développer un nouveau type d'appui aux collectivités à une échelle qui n'est pas celle des interventions habituelles des bureaux de conseil. Nonobstant les éventuels obstacles juridiques permettant d'établir ce type d'accompagnement impliquant plus d'un acteur public $^{20}$, nous tentons dans la section suivante de décrire les fondements (principes) et la méthodologie (processus et outils) qu'un tel accompagnement pourrait prendre.

\section{Une ingénierie territoriale propice à la coordination des organisations}

En s'inspirant des résultats de notre recherche, mais également d'autres expériences que nous avons conduites lors d'interventions telles que des prestations de conseils, expertises et projets de recherche auprès ou avec d'autres collectivités territoriales et services de l'État, nous proposons les bases d'une ingénierie territoriale (soit la combinaison de principes et d'une méthodologie) dont l'objectif est de renforcer la coordination inter-organisationnelle.

\footnotetext{
${ }^{20}$ Nous pouvons d'ores et déjà supposer qu'une telle mission d'accompagnement, si elle était réalisée par un prestataire externe, ne pourrait s'opérer dans le cadre classique d'une procédure d'appel d'offres, mais devrait plutôt suivre une forme plus souple de partenariat. Le code des marchés publics prévoit par exemple des contrats de mandat entre personnes publiques qui pourraient constituer une solution à explorer.
} 
Cette ingénierie ne cherche pas à créer de nouvelles procédures ou normes figées, mais plutôt à accompagner les personnes dans un processus de collaboration plus souple et adaptatif qui s'affranchit des contraintes qui ne peuvent être dépassées par les mécanismes formels habituels. La logique sous-tendant cette ingénierie porterait ainsi sur le renforcement des capacités des différents acteurs à se comprendre, à construire un projet commun et, enfin, à mettre en place les apprentissages nécessaires (Chia et al., 2008).

\subsection{Quelques principes transversaux}

La mise en place d'une coordination inter-organisationnelle repose sur une somme d'interactions entre des personnes. L'attitude de ces personnes constitue une caractéristique primordiale qui peut modifier la teneur de la coordination. Sans chercher à comprendre la complexité des processus intérieurs qui induisent ces attitudes, nous identifions néanmoins quelques principes (ou configurations) capables d'influencer d'une façon positive la qualité des interactions interpersonnelles nécessaires à la construction d'une coordination. Ces principes composent la logique de l'ingénierie proposée. Ils touchent autant des aspects individuels que collectifs.

Premièrement, la motivation individuelle est primordiale pour qu'un groupe de personnes s'engage dans une coordination. Elle nécessite l'identification d'un ou plusieurs avantages conférés à chaque individu par cette coordination (p.ex. travailler d'une façon plus efficiente, atteindre ses objectifs, participer à la prise de décision, etc.). Mais ces avantages individuels ne sont pas les seuls en jeu. Une coordination bénéficie également de la construction d'avantages mutuels à collaborer (p.ex. éviter des conflits futurs) qu'il convient de formaliser à travers un projet commun.

Deuxièmement, une coordination inter-organisationnelle implique la rencontre de personnes ne partageant pas forcément le même langage ou la même compréhension du problème. Afin de rendre possibles les échanges et d'objectiver les positions de chacun, il est important d'établir une représentation partagée du système. En co-construisant cette représentation, on dissocie alors la partie objectivable du problème (les faits sur lesquels on s'accorde) des intérêts propres à chaque acteur ou organisation qu'il représente.

Troisièmement, pour que des organisations collaborent dans la construction d'une action publique, elles doivent reconnaître la légitimité des différentes parties à aborder ou traiter cette action. Primordiale, cette reconnaissance n'implique pas forcément de dépasser les compétitions ou clivages existants, mais de les acter et de les accepter comme inhérents d'un système institutionnel complexe dans lequel les compétences et les responsabilités sont partagées. Cette reconnaissance repose aussi sur le principe de confiance vis-à-vis de la sincérité des autres parties, confiance pouvant être favorisée en rendant transparentes les attentes, contraintes et marges de manœuvre des différentes organisations et personnes vis-à-vis de la problématique traitée.

Quatrièmement, les personnes s'engageant dans une coordination inter-organisationnelle doivent également accepter un certain degré d'incertitude, tant au niveau des résultats de la démarche, qu'au niveau des connaissances qu'ils apportent, qui ont potentiellement vocation à être discutées ou remises en question par les autres. La coordination implique donc un processus d'apprentissage individuel et collectif qui remet en question les certitudes des uns, et le positionnement des autres. Les individus doivent être capables 
de constater et d'admettre les limites du fonctionnement institutionnel en place, afin de le questionner. Ils doivent également être capables d'élargir leurs champs du possible afin d'explorer de nouveaux modes opératoires et identifier des alternatives pertinentes.

\subsection{Une méthodologie participative}

Nous proposons ici une méthodologie participative qui suit la logique précédemment exposée. Nous décrivons différentes étapes permettant de renforcer la coordination entre des organisations autonomes mais concernées par un problème commun, et cherchant in fine à renforcer la gouvernance territoriale.

\section{Préciser les contours du besoin de coordination et initier le dialogue}

Nous partons de l'hypothèse que la mise en place d'un processus visant à renforcer la gouvernance territoriale doit nécessairement s'ancrer sur un problème spécifique qui légitime la construction d'un dialogue entre plusieurs organisations. Ce problème doit être ressenti comme tel, et associé à un besoin de changement dans les modes de gestion en place. En général, ce besoin émane d'un nombre restreint d'acteurs avec lesquels débute la démarche. Afin de préciser les contours du besoin de coordination et initier le dialogue, plusieurs étapes peuvent être suivies :

\section{a. Cerner les limites physiques du problème}

Nous proposons dans un premier temps d'analyser les phénomènes en jeu et voir quel périmètre physique est pertinent pour les traiter. Ce périmètre correspond au territoire d'action, soit la zone géographique sur laquelle s'ancre le problème initial. Même s'il a la possibilité d'évoluer en cours de processus, ce périmètre doit être caractérisé pour initier la démarche.

\section{b. Comprendre le jeu d'acteurs}

Il s'agit de définir les personnes concernées par le problème et leur position vis-à-vis de ce dernier, mais aussi vis-à-vis du principe de coordination. Un outil utile est « l'analyse interactive du jeu d'acteurs » qui permet de visualiser l'ensemble des acteurs et de leurs influences respectives dans un tableau à deux dimensions (pouvoir/intérêt), puis de faire ressortir leur position vis-à-vis de la mise en place d'une démarche de coordination interorganisationnelle (concernant, défavorable, neutre) (von Korff et al., 2011). Cet exercice interactif peut être conduit pour initier une discussion au sein d'un groupe restreint afin de faire ressortir les organisations et personnes à impliquer dans la suite du processus.

\section{c. Identifier le(s) besoin( $(s)$ de coordination a priori}

L'identification du ou des besoins peut être réalisée sur la base des résultats des étapes précédentes qui identifient le territoire pertinent et incluent les acteurs dont les actions conditionnent le phénomène ou qui sont impactés par celui-ci. Un travail de « brainstorming » peut être conduit avec le groupe restreint d'acteurs pour identifier le(s) besoin(s) de chaque organisation concernant la mise en place d'une coordination. En approfondissant ces besoins, on découvre qu'ils sont parfois associés à d'autres intérêts qui peuvent questionner la pertinence du problème initial. Ce dernier, qui constitue la porte d'entrée, pourra donc évoluer au cours de la démarche. 


\section{d. Légitimer un porteur de la démarche}

En fonction des résultats des étapes précédentes, un responsable de la démarche doit pouvoir se démarquer. Son choix repose avant tout sur sa motivation et sa légitimité, mais il doit aussi être associé à des compétences spécifiques. Le plus souvent le porteur de la démarche n'aura pas de pouvoir sur les autres partenaires. Cette idée du chef de fil sans pouvoir hiérarchique est développée par d'autres auteurs (Vanier 2008). Néanmoins, il doit être reconnu par les partenaires pour s'emparer de l'organisation du processus de mise en réseau et de structuration de la gouvernance. Son rôle n'est donc pas hiérarchique, il ne va pas chercher à imposer sa vision aux autres, mais il va plutôt activer le processus, générer la mise en relation entre les organisations et les personnes, être le gardien du temps en veillant aux échéances. C'est donc aussi un médiateur qui va développer un réseau (Moss 2009). Concrètement le porteur de la démarche peut être un binôme (élu-technicien), l'un donnant l'élan politique et la légitimité, l'autre étant en capacité de passer du temps à actionner les réseaux et organiser les rencontres. Le rôle des facilitateurs, sur lequel nous reviendrons plus loin, est différent.

\section{Appuyer l'ouverture et la compréhension mutuelle}

À ce stade, le groupe restreint doit être élargi aux organisations identifiées précédemment et qui seraient a priori favorables à la mise en place d'une coordination entre elles. En fonction du contexte, il est aussi possible d'impliquer des organisations qui seraient $a$ priori défavorables à la mise en place d'une coordination, mais dont l'intérêt serait grand vis-à-vis du problème dans la mesure où (i) l'analyse initiale pourrait s'avérer erronée, (ii) ces organisations pourraient évoluer en cours de processus, et (iii) elles pourraient bloquer celui-ci si elles n'étaient pas impliquées. Le recours à un facilitateur externe, qui n'a pas d'intérêt par rapport au problème, est à présent essentiel. Ce professionnel des méthodes de participation a pour mission d'appuyer le processus, d'une façon neutre et efficace. Il ne se substitue en aucun cas au porteur précédemment cité.

\section{a. S'accorder sur un avantage mutuel}

Cette étape consiste à élargir la prise de conscience de l'intérêt réciproque des différentes organisations à collaborer sur le problème identifié. La restitution du travail initial réalisé par le groupe restreint auprès des autres organisations peut servir de base à un débat dont l'objectif est de faire ressortir les traits d'un avantage mutuel qui dépasse la simple somme des intérêts particuliers. La rencontre donne également l'occasion de présenter la démarche et acter les engagements des différentes organisations dans celle-ci. S'agissant d'une rencontre élargie faisant intervenir des organisations pouvant appartenir à différents systèmes institutionnels, la facilitation des échanges est essentielle car elle permet une compréhension mutuelle et la construction progressive d'un langage partagé (Kaner et al., 1996).

\section{b. Construire une représentation partagée du système}

Étape essentielle, la construction d'une représentation partagée du système (ou du problème) entre les personnes impliquées dans le processus permet d'ancrer le dialogue sur un objet factuel (la représentation du système), et donc de forcer la formalisation des positions et l'objectivation des arguments. Le but n'est pas d'unifier les points de vue, mais de permettre de les formaliser sur un support commun. Plusieurs outils peuvent appuyer 
ce travail, notamment la modélisation ou la cartographie participative dont l'objectif est de dessiner collectivement les contours d'un système ou d'un territoire (von Korff et al., 2011). Outre la formalisation des aspects factuels, il est également possible d'interpréter leurs conséquences, et réaliser ainsi un diagnostic du problème.

\section{c. S'ouvrir aux différents systèmes institutionnels}

Cette étape n'est pas structurante, mais peut appuyer le bon déroulement du processus, notamment si les acteurs et organisations impliqués appartiennent à des systèmes institutionnels éloignés. Dans ce cas, un travail plus approfondi peut s'avérer nécessaire pour améliorer la compréhension réciproque des logiques institutionnelles, à savoir les règles et valeurs - formelles et informelles - qui guident les positions et actions des différentes organisations. Les jeux de rôles sont d'excellents outils pour permettre aux uns de se projeter à la place des autres, et ainsi dépasser les cloisonnements habituels (Tsuchyia, 1998).

\section{Identifier des alternatives afin d'améliorer la gouvernance en place}

Lorsque les conditions du dialogue sont réunies, que les organisations et personnes impliquées s'accordent sur un avantage mutuel, qu'elles partagent des éléments de langage, qu'elles ont construit une représentation commune du système, et qu'elles acceptent les systèmes institutionnels en jeu, les différentes organisations sont alors prêtes à rechercher des alternatives. Cette étape repose sur la créativité des participants et l'intelligence collective qui résulte de leurs échanges. Mais elle peut également reposer sur l'analyse de solutions développées dans d'autres contextes. La recherche d'alternatives peut porter sur les différents aspects de la gestion de la ressource ou du problème d'intérêt général sur lequel la coordination inter-organisationnelle est ancrée, notamment :

- la production et l'échange d'informations sur le système considéré;

- l'évaluation de l'état du système en lien avec des objectifs précis;

- le mécanisme de prise de décision collective en lien avec la planification d'actions ou de processus spécifiques;

- le système de règles, de contrôle et de sanctions.

En fonction des aspects traités, il peut être nécessaire de déployer des outils et des compétences spécifiques, en faisant notamment appel à une expertise complémentaire. À titre d'exemple, il est possible d'évaluer l'acceptabilité sociale de nouvelles règles ou sanctions en les testant d'une façon virtuelle. Ces tests peuvent prendre diverses formes telles que les mises en situation, le théâtre forum ou encore les exercices de simulation politique. D'autres aspects, comme l'évaluation de l'état du système, nécessitent le déploiement d'outils différents, tels que les analyses multicritères ou d'autres grilles d'analyse et d'évaluation. Mais la caractéristique commune de ces outils est qu'ils doivent pouvoir être déployés au sein d'un groupe, et doivent donc nécessairement intégrer une interface interactive ou un usage participatif.

\section{Formaliser et opérationnaliser les changements souhaités}

Nous avons décrit jusqu'ici un processus d'innovation collective et d'exploration de solutions de coordination. Vient ensuite la mise en œuvre qui nécessite d'ancrer de nouvelles routines et de nouvelles manières de travailler. La consolidation des principes de coordination élaborés nécessite alors un retour vers la formalisation et vers l'opération- 
nalisation. Mais formaliser des accords, des actions, des procédures avec plus de trois organisations devient vite compliqué et long. L'étape de mise en œuvre nécessite donc de redescendre à une échelle plus restreinte, afin de renégocier des accords (souvent bilatéraux) entre les acteurs concernés. Le résultat du processus doit être de changer la perspective de ces contrats ou engagements bilatéraux : ces accords formalisés doivent tenir compte des interdépendances et des connaissances collectives générées dans le processus. $\mathrm{Au}$ final, ces accords doivent permettre de prendre en considération les effets potentiels des actions qu'ils encadrent sur les tiers.

\section{Conclusion}

La gouvernance territoriale traduit d'importantes transformations de l'action publique dont la mise en œuvre ne repose plus uniquement sur un modèle central et descendant, mais est complétée par des modes de coordination plus horizontaux, souples, dynamiques et intégratifs. Ces modes de coordination renvoient généralement aux différents processus de concertation permettant d'impliquer les acteurs locaux (usagers, citoyens, etc.) dans la construction de l'action publique. Tout d'abord, nous démontrons que lorsque le problème dépasse les périmètres de compétences strictes d'un acteur public sans être précisément cadré au niveau réglementaire, la gouvernance territoriale passe avant tout par une meilleure coordination entre les organisations concernées par ce problème. $\mathrm{Si}$ ce modèle semble séduisant, l'analyse de terrain révèle cependant une multitude de contraintes vécues au quotidien par les différentes organisations devant se coordonner. Notre étude de cas sur la gestion des ressources en eau entre les bassins de l'Orb et de la Basse Vallée de l'Aude illustre certaines d'entre elles. Nous avons ainsi abordé des difficultés relatives aux périmètres d'intervention des organisations, à l'articulation entre les outils de planification et ceux de gestion opérationnelle, et aux divergences politiques. Nous montrons également que ces freins à la coordination n'agissent pas avec la même intensité sur les différentes organisations, en raison de différences liées à leur environnement institutionnel et aux capacités et positions des personnes qui les composent. Enfin, bien qu'un besoin spécifique ait été identifié, nous révélons qu'il n'existe pas, à l'heure actuelle, d'offre d'accompagnement à la coordination inter-organisationnelle. Si l'on souhaite renforcer la gouvernance territoriale, il est donc nécessaire de penser et concevoir une nouvelle forme d'ingénierie territoriale.

En formalisant certains principes ainsi que différentes étapes d'une méthodologie participative, nous tentons alors de démontrer comment il est possible d'accompagner la coordination inter-organisationnelle. Cette ingénierie territoriale est avant tout un instrument de dialogue et d'apprentissage. En effet, la méthodologie proposée repose sur le développement de conditions propices à la compréhension mutuelle, la recherche d'un avantage mutuel à collaborer, la création d'un langage partagé et d'une représentation commune du système, la reconnaissance des différents systèmes institutionnels en jeu et, enfin, la recherche d'alternatives au problème rencontré. Elle ne vise donc pas à créer un nouveau niveau de complexité administrative, ni à se substituer aux procédures formelles découlant de la réglementation. L'ingénierie proposée cherche en revanche à accompagner les organisations dans leurs choix lorsque ces procédures formelles ne sont plus suffisantes. Elle s'appuie pour cela sur des apprentissages individuels (p.ex. en renforçant 
la compréhension des interdépendances d'un système) mais aussi sur des apprentissages sociaux (p.ex. en augmentant la capacité d'un groupe de personnes à se comprendre).

En croisant une approche analytique avec un travail d'expertise, nous avons donc dressé les contours d'une ingénierie qui répond aux enjeux de ces nouvelles formes d'action publique. Si elle n'ôte en rien la nécessité de conserver des procédures d'arbitrage de l'État (notamment lorsque les intérêts des différentes organisations sont trop antagonistes), cette ingénierie questionne cependant la façon dont les acteurs publics font appel à l'appui d'un tiers. Il convient à présent de se pencher sur l'ancrage administratif de ce type de processus qui, par sa souplesse, son adaptabilité, sa conduite collective, relève plus du partenariat que de la prestation de conseil. La place de la recherche, mais aussi des bureaux d'études, gagnerait ainsi à être reconsidérée afin de créer des espaces dont la finalité n'est pas la définition de la meilleure solution, mais la mise en place de conditions propices à l'apprentissage collectif. Au final, c'est cet apprentissage qui permettra de faire face aux défis actuels et futurs de la gestion des territoires et des ressources naturelles.

\section{Références}

Banque Mondiale, 1992. Governance and development. Washington DC, World Bank.

Baron C., 2003. La gouvernance : débats autour d'un concept polysémique. Droit et société 54 (2), 329-349.

Baron C. et Belardi W., 2010. Gouvernance participative et rôle des associations pour l'accès à l'eau dans la périphérie de Casablanca (Maroc) In G. Schneier-Madanes (Ed.), L'eau mondialisée : les enjeux de la gouvernance. La Découverte, Paris, 381-402.

Barraqué B., 1997. Subsidiarité et politique de l'eau / Subsidiarität und Wasserpolitik In A. Faure (Ed.), Territoires et subsidiarité, l'action publique locale à la lumière d'un principe controversé. L'Harmattan, Paris, 165-201.

Barret Ph., 2003. Guide pratique du dialogue territorial. Concertation et médiation pour l'environnement et le développement local. Fondation de France, Paris.

Becattini G., 1989. Sectors and/or Districts: some Remarks on the Conceptual Foundations of Industrial Economics In E. Goodman, J. Bamford et P. Saynor (Ed.), Small Firms and Industrial Districts in Italy, Routledge, London, 123-135.

Blondiaux L., 2008. Le nouvel esprit de la démocratie. Seuil, Paris.

Borrini-Feyerabend G., Farvar M. T., Nguinguiri J. C. and Ndangang V. A., 2000. Co-Management of Natural Resources: Organising, Negotiating and Learning-by-Doing. GTZ and IUCN, Kasparek Verlag, Heidelberg.

Borrini-Feyerabend G., Chatelain C., Hosh G., et al., 2010. En Gouvernance Partagée! Un guide pratique pour les aires marines protégées d'Afrique de l'Ouest, PRCM, UICN et CEESP, Dakar.

Brun A., 2010. Les contrats de rivière en France : un outil de gestion concertée de la ressource en local In G. Schneier-Madanes (Ed.). L'eau mondialisée : les enjeux de la gouvernance. La Découverte. Paris, 305-321.

Cattellin M., 2008. Modes de coordination, réseaux interorganisationnels et avantage concurrentiel : une application à une destination touristique des Alpes. Journée Rochelaise de Recherche sur le Tourisme. La Rochelle, France, Groupe Sup de Co.

Chambers R., 1991. Shortcuts and Participatory Methods for Gaining Social Information for Projects In Cernea, M. (Ed.). Putting People First. Sociological Variables in Rural Development, Oxford University Press, New York.

Chevalier J., 2003. L’État post-moderne, LGDJ, Paris.

Chia E., Torre A. et Rey-Valette H., 2008. Conclusion : Vers une « technologie » de la gouvernance territoriale ! Plaidoyer pour un programme de recherche sur les instruments et dispositifs de la gouvernance des territoires, Norois, http://norois.revues.org/2603

Creighton J. L., 2005. The Public Participation Handbook: Making Better Decisions through Citizen Involvement. Jossey-Bass, San Francisco. 
Crozier M. et Friedberg E., 1977. L'acteur et le système. Le Seuil, Paris.

David A., 2008. La recherche-intervention, cadre général pour la recherche en management? In David A., Hatchuel A. et Laufer R. (Ed.) Les nouvelles fondations des sciences de gestion, $2^{2}$ édition, Vuibert, Paris, 193-213.

Dufour D.-R., 2009. La gouvernance comme nouvelle forme de contrôle social. Connexions 91, 41-54.

Duran P. et Thoenig J-C., 1996. L'État et la gestion publique territoriale. Revue française de science politique 4, 580-623.

Edelenbos J., 1999. Design and Management of Participatory Public Policy Making. Public Management Review 1, 569-576.

Edelenbos J. et Teisman G. R., 2011. Numéro spécial sur la gouvernance de l'eau. Prologue. La gouvernance de l'eau? Les actions de l'État, entre la réalité de la fragmentation et le besoin d'intégration. Revue Internationale des Sciences Administratives 77 (1), 5-30.

Faure A. et Negrier E., 2007, Les politiques publiques à l'épreuve de l'action locale. Critique de la territorialisation. L'Harmattan, Paris.

Froger G., 2006. Significations et ambiguïtés de la gouvernance dans le champ du développement durable. Mondes en développement 136 (4), 11-28.

Ghiotti S., 2006. Les Territoires de l'eau et la décentralisation. La gouvernance de bassin-versant ou les limites d'une évidence, Développement durable et territoires, Dossier 6, http://developpementdurable.revues. org $/ 1742$

Guérin-Schneider L., Dionnet M., Abrami G., von Korff Y., 2010. Comment évaluer les effets de la modélisation participative dans l'émergence d'une gouvernance territoriale. Colloque OPDE: Aide à la décision et gouvernance, 25 au 26 octobre 2010. INRA, Montpellier http://hal.archives-ouvertes.fr/docs/00/58/40/48/ PDF/MO2010-PUB00030287.pdf

Jessop B., 1998. L'essor de la gouvernance et ses risques d'échec : le cas du développement économique. Revue internationale des Sciences Sociales 155, 31-49.

Kaner S., 1996. Facilitator's Guide to Participatory Decision-making. New Society Publishers, Canada.

Lardon S., 2007. Quelle ingénierie territoriale pour les territoires peri-urbains? Proposition d'une démarche In Loudiyi S., Bryant C. R., Laurens, L. Territoires périurbains et Gouvernance. Perspectives de recherche. Éditions du laboratoire développement durable et dynamiques territoriales, Université de Montréal, 149-158.

Lascoumes P. 1990. Normes juridiques et mise en œuvre des politiques publiques. L'Année sociologique 71.

Latour B. et Le Bourhis J. P., 1995. Donnez-moi de la bonne politique et je vous donnerai de la bonne eau... Rapport sur la mise en place des Commissions Locales de l'Eau, ENSMP, Paris.

Leloup F., Moyard F. et Pecqueur B., 2005. La gouvernance territoriale comme nouveau mode de coordination territoriale? Géographie, économie, société 7 (4), 321-332.

Meyer J. W. and Roman B., 1977. Institutionalized Organizations: Formal Structure as Myth and Ceremony. American Journal of Sociology 83 (2), 340-363.

Moquay P., Maurel P., Lardon S. et Piveteau V., 2001. Pour un usage raisonné des représentations spatiales : bilan et perspectives In Moquay P., Maurel P., Lardon S., Piveteau V. (Ed.), Représentations spatiales et développement territorial, Hermes Science, Paris, 411-437.

Moss T., 2009. Intermediaries and the Governance of Sociotechnical Networks in Transition. Environment and planning 46 (1), 1480-1495.

Narcy J.-B., 2000.Les conditions d'une gestion spatiale de l'eau. Thèse de doctorat Sciences de l'environnement, ENGREF, Paris.

Ostrom E., 1990. Governing the Commons: The Evolution of Institutions for Collective Actions. Cambridge University Press, Cambridge.

Papadopoulos Y., 2003. Gouvernance et transformations de l'action publique : quelques notes sur l'apport d'une perspective de sociologie historique In P. Laborier et D. Trom. (Ed.), Historicités de l'action publique, PUF, Paris, 119-135. 
Pasquier R., Simoulin V. et Weisbein J., 2007. La gouvernance territoriale. Pratiques, discours et théorie. L.G.D.J., Paris.

Petit O., 2004. La surexploitation des eaux souterraines : enjeux et gouvernance. Natures Sciences et Sociétés 12 (12), 146-156.

Rey-Valette H. et Antona M. 2010. Gouvernance et gestion intégrée du littoral : l'exemple des recherches francaises. Vertigo 9 (1).

Rey-Valette H., Pinto M., Maurel P., Chia E., Guihéneuf P.-Y., Michel L., Nougarèdes B., Soulard C., Jarrige F., Guillemot S., Cunnac M., Mathé S., Barbe E., Ollagnon M., Delorme G., Prouhèze N., Laurent A., Suita L.A., Bertrand J., Dieudonné C., Morvan R. et Champrigaud A., 2011. Guide pour la mise en cuvre de la gouvernance en appui au développement durable des territoires, Cemagref, CNRS, Geyser, Inra, Supagro, Université Montpellier 1. Diffusion INRA-Montpellier.

Ring P. S. and van de Ven, A.H., 1994. Developmental Processes of Cooperative Interorganizational Relationships. The Academy of Management Review, 19 (1), 90-118.

Riveline C., 1991. Un point de vue d'ingénieur sur la gestion des organisations. Annales des Mines. Gérer et Comprendre (décembre), 50-62.

Rowe G. and Frewer L. J., 2004. Evaluating Public Participation Exercices. A Research Agenda. Science, Technology \& Human Values 29 (4), 512-556.

Schmitt M., Grégoire B. and Dubois E., 2005. A Risk Based Guide to Business Process Design in InterOrganizational Business Collaboration. International Workshop on Requirements Engineering for Business Need and IT Alignment, Paris, August 2005. http://efficient.tudor.lu/cms/efficient/content.nsf/0/4A93885 2840437F2C12573950056F7A9/\$file/SchmittGregoireDubois_Efficient_RiskBasedBmBpmAlignment_ REBNITA05.pdf.

Stoker G., 1998. Governance as Theory: Five Propositions. International Social Science Journal 50 (155), 17-28.

Théret B., 1998. La régulation politique : le point de vue d'un économiste In J. Commaille et B. Jobert (Ed.), Les métamorphoses de la régulation politique, LGDJ, Paris, 83-118.

Tsuchyia S., 1998. Simulation/Gaming as an Essential Enabler of Organizational Change. Simulation \& Gaming 29 (4), 400-408.

Vanier M., 2008. Le pouvoir des territoires - Essai sur l'interterritorialité. Economica, Paris.

Von Korff Y., Dionnet M., Imache A., Geney C., Rougier J.-E., Leteurtre E. et Dolinska O., 2011. Manuel de Concertation Territoriale. Lisode, Montpellier.

Xhauflair V., Pichault F. et MaesschalckM., 2010. Partenariats inter-organisationnels et nouvelles formes de gouvernance : les conditions d'un compromis équilibré et pérenne. Management \& Avenir, 33 (3), 298-316. 